\title{
NARROW BAND INTERFERENCE FILTER FOR THE IR-SPECTRUM REGION
}

Iryna Ya. Yaremchuk, Volodymyr M. Fitio, Yaroslav V. Bobitski Department of Photonics, Lviv Polytechnic National University 12 Bandera Str., 79013 Lviv, Ukraine

Phone: 8-032-2582581, e-mail: yaremchuk@polynet.Iviv.ua

\section{Introduction}

Recently, the synthesis of the multi-layered optical systems gained large enough development, successes of these researches influence both on development of optics and other branches of science and technique. Presently, it is possible to get the interference filters of any type for all of optical range with the set spectral descriptions. To the one of the most topical problems now is design of narrow-band filters in optical instrument engineering, as industry of application is very wide, this is astrophysics, chemistry, medicine, materials science, engineering [1 - 3].

As is generally known, one of basic characteristics of narrow-band filters is consider the size of sidebands suppression (attenuation range) - size of spectral interval in which a transmission is low $(0.1 \%)[4]$. However, it is enough difficultly to synthesize the interference narrow-band filter with the wide sidebands suppression without substantial losses for a working wavelengt. Traditionally this problem is decided the using additional short-wave and long-wave filters [5]. This complicated the construction of filter substantially increased absorption, and also decreased of transmission for working wavelength. All of it resulted in an attempt to construct or optimize a filter so that to sidebands suppression of transmission and get the curve of transmission without the substantial losses of absorption for working wavelength.

In papers [6 - 7] we offered the structure of the narrow-band interference filter, that consisted only of five layers. In this filter substrate was basis of filter and simultaneously the basic layers of all of the multi-layered system of type $\mathrm{HL} 500 \mathrm{H} \mathrm{LH}$. Where $\mathrm{H}$ and L layers of materials with high $\left(n_{H}=4.01\right)$ and low $\left(n_{L}=1.44\right)$ indices refractions accordingly. The optical thicknesses layers are equal quarterwavelength, size 500 means that the optical thickness of separate layer is equal $500 \lambda_{0} / 4\left(\lambda_{0}\right.$ - working wavelength). Such filter can be designed on any wave-length. The curve of transmission such filter for the working wave-length $3,85 \mu \mathrm{m}$ is represented on the fig. 1 . But the spectral characteristic contains the close located sidebands maximums.

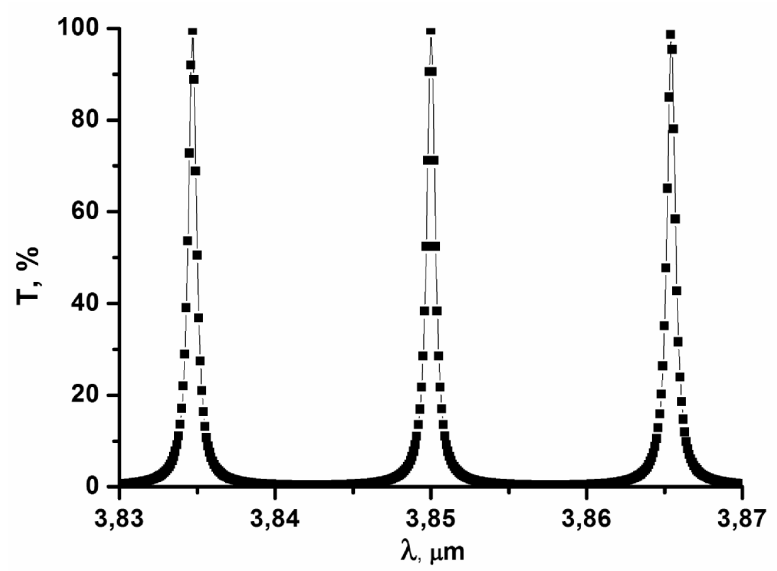

Fig. 1. The dependence of transmittance from wavelength for filter of type $\mathrm{HL} 500 \mathrm{H} \mathrm{LH}$

$$
n_{H}=4.01, n_{L}=1.44, \lambda_{0}=3.85
$$

In this work optimization of structure of narrow-band filter is conducted with the purpose of improvement of selective properties due to suppression of sidebands maximums of transmission with the minimum losses of absorption for working wavelength. The calculations by a matrix method were performed [8]. 


\section{An additional interference filter is for sidebands suppression}

Additional filter that is deposited on the base construction of the narrow-band filter is offered for sidebands suppression of transmission. The decision of the put problem is executed in two stages:

- design of additional narrow-band filter, basic transmission peak must check with the maximum of transmission of basic filter for working wavelength, pass band width must be no more distance between two sidebands of basic filter. Sidebands maximums of transmission additional filter must not check with sidebands maximums of transmission basic filter;

- concordances these two filters through a transition layer with the purpose of receive of the given spectral curve.

It is the problem to design the additional narrow-band interference filter for sidebands suppression of transmission of filter of type $\mathrm{HL} 500 \mathrm{H} \mathrm{LH}$, that has the basic transmission peak on working wavelength $\lambda_{0}=3.85 \mu \mathrm{m}$ and pass band width $\Delta \lambda \leq 0.02 \mu \mathrm{m}$, because filter with the more value $\Delta \lambda$ will be pass of the sidebands of transmission.

As is generally known, the simplest variant of narrow-band filter is the filter with the structure similar to the standard Fabry-Perot [9]. This filter includes interference mirrors, which consist of layers with high and low indeces refractions of identical optical thickness that equal quarter-wavelength. This mirror is separated the layer with the high index of refraction the optical thickness of which is multiple half wavelength. The same materials as for the synthesis of base filter it are $\mathrm{Ge}$ and $\mathrm{SrF}_{2}$ with the indeces of refraction $n_{H}=4.01$ and $n_{L}=1.44$ by absorptances $0.02 \mathrm{sm}^{-1}$ and $0.001 \mathrm{sm}^{-1}$ accordingly [10] are used for the calculation of transmission of additional filter.

The optimal amount of layers in the system and thickness of dividing layer are used for achievement of the given bandwidth of transmission. The pass band was wide enough (region of wavelengths is from $3.835 \mu \mathrm{m}$ to $3.865 \mu \mathrm{m}$ and transmission anymore after $10 \%$ ) (see fig. 2 (curve 1)) when used the structure from 9 layers. During the use of such additional filter the optimized filter will be pass not only the basic pass band but also sidebands.

It is possible narrowing pass band due to the increase of thickness of middle layer, however given such step was not by the desired result (see fig. 2, curve 2). Use 13 layers in the system and thicknesses of middle layer equal half of wavelength gave necessary us pass bandwidth and zero of transmission after its limits (see fig. 2, curve 3).

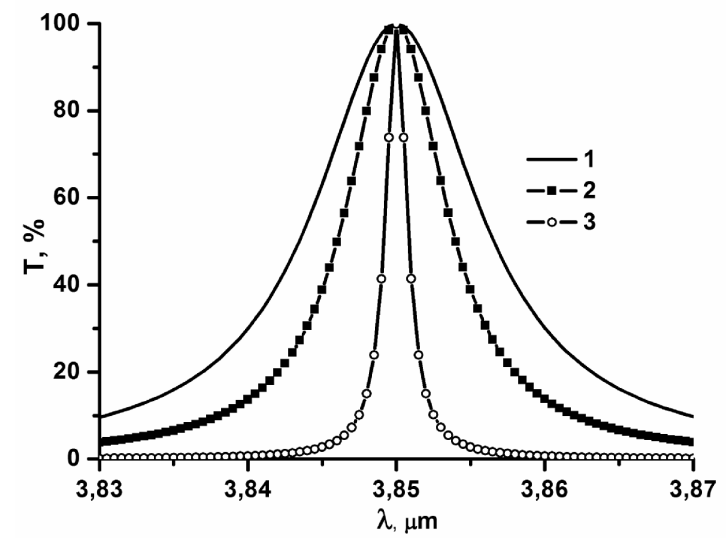

Fig 2 The dependence of transmittance from wavelength for additional narrow-band filter of type $(\mathrm{HL})^{2} 2 \mathrm{H}(\mathrm{LH})^{2}-$ curve $1 ;(\mathrm{HL})^{2} 4 \mathrm{H}(\mathrm{LH})^{2}-$ curve $2 ;(\mathrm{HL})^{3} 2 \mathrm{H}(\mathrm{LH})^{3}$ - curve 3.

Usually the pass band can be yet narrowed increasing the optical thickness of middle layer, however much the system $(\mathrm{HL})^{3} 2 \mathrm{H}(\mathrm{LH})^{3}$ fully answers the put requirements to the additional interference narrow-band filter, that is why in subsequent calculations used exactly it.

\section{A narrow-band interference filter is optimized}

If on a base narrow-band filter to deposited the additional filter we not only will not sidebands suppressed maximums of transmission but also yet will approach them to basis. For such connection an top layer of material with the high index of refraction of basic filter and first layer of additional filter form another separate layer with the high index of refraction. To connection these two filters, is used the transitional layer with the low index of refraction. This layer will be eliminate creation of additional resonance layer. The spectral curve represented on a fig. 3 depends on the choice of thickness of this 
transitional layer. The sidebands maximums of transmission are suppressed (see fig. 3, curve 2) when the optical thickness of transitional layer is equal quarter-wavelength.

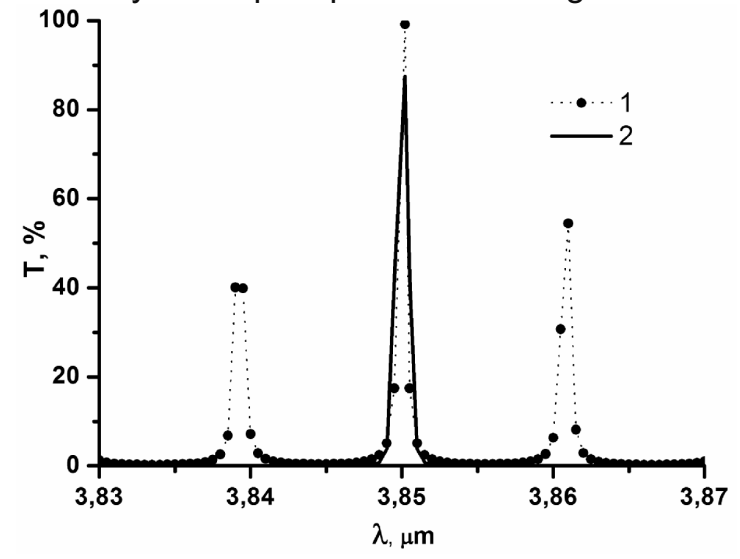

Fig. 3 The dependence of transmittance from wavelength for filter of type $\mathrm{HL} 500 \mathrm{H} \mathrm{LH} 4 \mathrm{~L}(\mathrm{HL})^{3} 2 \mathrm{H}(\mathrm{LH})^{3}$ - curve 1; HL 500H LH L (HL) ${ }^{3} 2 \mathrm{H}(\mathrm{LH})^{3}$ - curve 2.

The spectrum of transmission of the optimized structure is represented on the fig. of $4 \mathrm{a}$. The curve of transmission contains a dip on a working wavelength (see fig. of $4 \mathrm{~b}$ ); however the transmission is $87 \%$ at the account of absorption in materials that answers requirements to such type of filters.
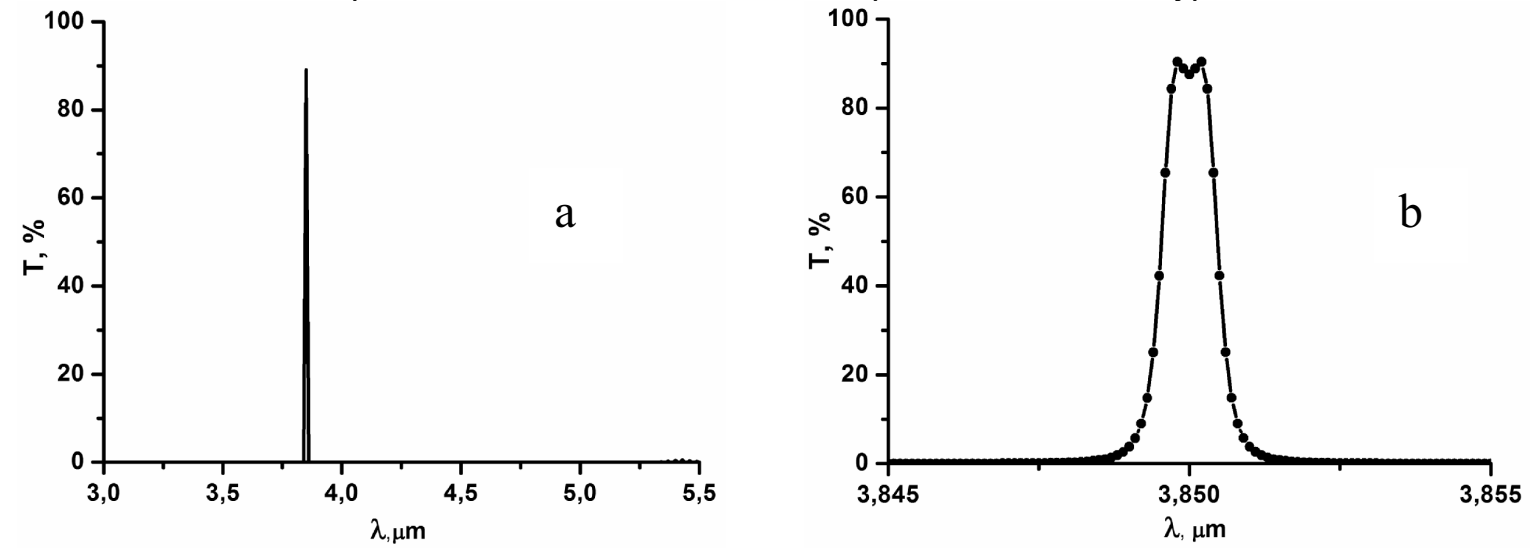

Fig. 4 The dependence of transmittance from wavelength for filter of type $\mathrm{HL} 500 \mathrm{H} \mathrm{LH} \mathrm{L}(\mathrm{HL})^{3} 4 \mathrm{H}(\mathrm{LH})^{3}$

It is needed to mark that the optical thickness of transitional layer can be used greater after $\lambda_{0} / 4$, for example $3 \lambda_{0} / 4$, however the curve of transmission will be deformed (see fig. 5).

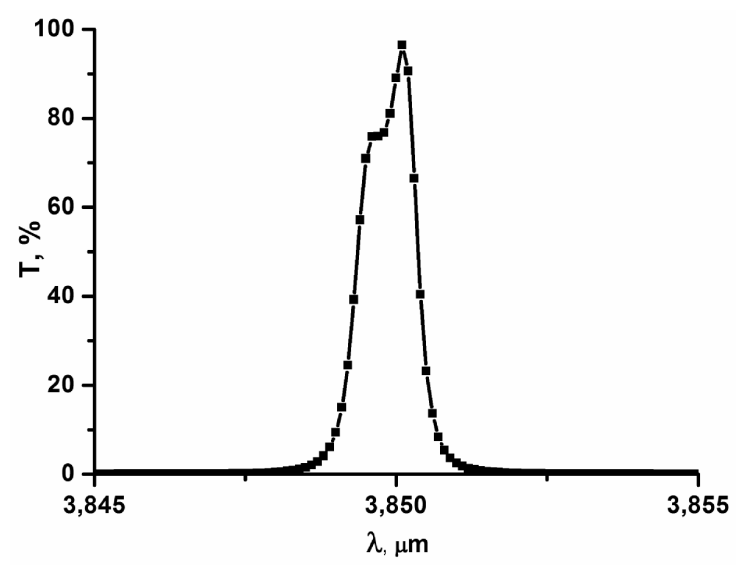

Fig. 5 The dependence of transmittance from wavelength for filter of type $\mathrm{HL} 500 \mathrm{H} \mathrm{LH} 3 / 4 \mathrm{~L}(\mathrm{HL})^{3} 2 \mathrm{H}(\mathrm{LH})^{3}$ 


\section{Conclusion}

The method of sidebands suppression of maximums of transmission without the use additional cutoff filters is offered in this work. The problem is decided application of structure, which include the additional narrow-band filter connected with basic by the transitional layer. The eventual structure of optimized filter has the appearance of $\mathrm{HL} 500 \mathrm{H} \mathrm{LH} \mathrm{L}(\mathrm{HL})^{3} 4 \mathrm{H}(\mathrm{LH})^{3}$. In this interference filter sidebands maximums are suppressed on the region from $2.7 \mu \mathrm{m}$ to $5.7 \mu \mathrm{m}$, and pass band width is equal $0.002 \mu \mathrm{m}$. The transmission is equal to $87 \%$ on the working wavelength at the account of absorption by materials. Such filter can be modeled for any wavelength in the infra-red of spectrum. Optimization of structure allowed to sidebands suppressed maximums of transmission and gets the curve of transmission without the substantial losses of absorption for working wavelength.

\section{References}

[1] Kong S.H., et al.//STW. - 1998. -10. - P. 291-294.

[2] Erdogan T., Mizrahi V.// Spectroscopy. - 2004. - 19 (12). - P. 113-116.

[3] Li. L. // Optics and Photonics News. - 2003. -9. - P. 24-30.

[4] Photooptic-Filters. www.photooptic-filters.com

[5] Optical coatings Japan. www.ocj.co.jp.

[6] Pat. 26850 Ukraine, G02B 5/28. Interference filter / I.Ya. Yaremchuk, V.M. Fitio, Ya.V. Bobitski. № u 200705597. - 4 p.

[7] I.Ya. Yaremchuk, V.M. Fitio, Ya.V. Bobitski // Sem. Phys. Quant. Elect. Optoel. - 2008. - 11 (1). P. 23-25.

[8] B. Maitland, M. Dunn. Laser Physics. - North-Holland Publishing Company, Amsterdam-London, 1969.

[9] N. Borisevich, V. Vereschagin, M. Validov. Infrared filters. - Minsk: Nauka i texn., 1971

[10] CRYSTAL Gmbn, Ostendstrabe 2-14, D-12459 Berlin, Germany, www.crystal-gmbn.com. 